\title{
El derecho a la educación y la seguridad en tiempos de Covid-19: Factores claves para la adopción de modelos de blended learning en centros de educación no universitaria en España
}

\author{
The right to education and security in Covid-19 times: Key factors \\ for the adoption of blended learning models in non-university \\ education centers in Spain
}

\author{
Amaya Gil Albarova \\ Universidad de Zaragoza (España) \\ ORCID: https://orcid.org/0000-0002-4756-4853
}

agilalbarova@unizar.es

\begin{abstract}
NOTA BIOGRÁFICA
Doctora en Sociología por la Universidad de Zaragoza. Colaboradora del Grupo de Investigación Sociedad, Incertidumbre y Creatividad (SIC). Cuenta con postgrados de especialización en los ámbitos de la docencia y gestión en Educación Superior. Desde 1988 ha ejercido su actividad docente e investigadora en diferentes centros y ha ocupado puestos de dirección y gestión universitaria en los últimos años.
\end{abstract}

Cristina Monge Lasierra

Universidad de Zaragoza (España)

ORCID: https://orcid.org/0000-0001-9972-6028

crismonge1@gmail.com

\section{NOTA BIOGRÁFICA}

Politóloga y doctora por la Universidad de Zaragoza, donde elaboró su tesis doctoral sobre la idea y práctica de participación en el movimiento del 15-M. Máster en Unión Europea por la UNED, Postgrado en participación ciudadana por la Universidad de Zaragoza, Máster en comunicación política por la Universidad Autónoma de Barcelona, y experta en función gerencial de ONGs por ESADE. Profesora de Sociología en la Universidad de Zaragoza y tutora de Sociología y Ciencia Política en la UNED, es investigadora asociada del instituto de gobernanza global Globernance y colabora en centros de formación como el INAP y en estudios de postgrado de distintas universidades. Miembro del Grupo de Investigación Sociedad, incertidumbre y creatividad. Es asesora ejecutiva de ECODES, miembro del consejo asesor de la Fundación Renovables y del capítulo español del Club de Roma. Analista política en distintos medios de comunicación (EI País, Infolibre, Cadena SER y TVE principalmente) y miembro del consejo editorial de Ethic. En "Agenda Pública" coordina la sección de "Transición Energética". Es miembro del Consejo de gobierno abierto del gobierno de España dependiente del ministerio de política territorial, del Consejo asesor de evaluación del Plan Nacional de adaptación al cambio climático del ministerio para la transición ecológica, y del Consejo nacional de cooperación al desarrollo, dependiente del ministerio de asuntos exteriores. Es autora de la monografía "15M: Un movimiento político para democratizar la sociedad" (PUZ, 2017), que recoge buena parte de su tesis doctoral, coautora de "La Iniciativa Social de Mediación en los conflictos del agua en Aragón" (PUZ, 2019) y co-editora de la colección "Más Cultura Política, Más Democracia" (Gedisa), en la que además ha publicado "Hackear la Política" (2019). Sus líneas de interés son la crisis ambiental, la Agenda 2030 como marco global para alcanzar la sostenibilidad, y la calidad democrática, fundamentalmente en lo referente a gobierno abierto y democracia deliberativa. 


\title{
NOTA BIOGRÁFICA
}

Doctora en sociología por la Universidad Pontificia de Salamanca en Madrid en 2003, desarrolla investigación inicialmente sobre demografía, turismo y desarrollo rural. Posteriormente se ha redirigido hacia la educación y nuevas tecnologías y hacía el estudio de la vulnerabilidad y mercado laboral en sus vertientes. Compatibilizo ejercicio profesional con docencia universitaria en la Universidad de Zaragoza desde 1999.

\author{
Federico Buyolo García \\ Ministerio de Educación y Formación Profesional (España) \\ ORCID: https://orcid.org/0000-0001-6427-5095 \\ federico.buyolo@educacion.gob.es
}

\section{NOTA BIOGRÁFICA}

Licenciado en Pedagogía. Master Universitario en Investigación e Innovación Educativa. Experto Universitario en estadísticas e indicadores educativos. Actualmente Doctorando en Teoría de la Educación por la UNED. Concejal del Ayuntamiento Elche entre los años 2003 y 2011. Diputado Nacional en la X Legislatura. Director General de Cooperación y Solidaridad de la Generalitat Valenciana de 2015 a 2018. Director General Oficina Alto Comisionado para la Agenda 2030 de 2018 a 2020. Actualmente Director Adjunto del gabinete de la Ministra de Educación y Formación Profesional de España.

\section{RESUMEN}

Este trabajo tiene como objetivo identificar los factores críticos de éxito en el diseño e implementación de programas de blended learning en las escuelas españolas como medio para conciliar el derecho a la educación y la seguridad sanitaria en tiempos de pandemia. Para ello, se empieza por trazar un semblante de algunos de los aspectos más significativos que describen la realidad educativa en centros españoles, para a continuación describir los diferentes escenarios que se plantean para retomar la actividad educativa a corto plazo. Una vez descrito el contexto, se sintetizan las principales características del blended learning, haciendo énfasis en los distintos modelos posibles. Para ello, se ha realizado una revisión de la literatura especializada que muestra evidencias empíricas sobre las experiencias ya implementadas en otros países. Con esa información, se identifican los factores que pueden ayudar a tomar decisiones en la implementación de estos programas, entre los que se encuentran algunos de carácter transversal y otros específicos sobre el modelo concreto por el que optar.

\section{PALABRAS CLAVE}

Desigualdades socioeducativas; estrategias de superación; Covid-19; blended learning; presencialidadvirtualidad; mediación tecno-pedagógica.

\begin{abstract}
This paper aims to identify the critical success factors in the design and implementation of blended learning programs in Spanish schools reconciling the right to education and health security in times of pandemic. It begins by tracing a semblance of some of the most significant aspects that describe the educational reality in Spanish centers to describe the different factors that arise to resume educational activity in the short term. Once the context is described, the main characteristics of blended learning are synthesized, emphasizing the different possible models, reviewing specialized literature that shows empirical evidence on experiences and implementations in other countries. Finally, with this information, factors that can help make decisions in the implementation of these programs are identified, distinguished the ones of transversal nature, and others specific to choose from.
\end{abstract}


GAPP. Nueva Época - N. ${ }^{\circ} 26$, julio 2021 - ISSN: 1989-8991 - DOI: https://doi.org/10.24965/gapp.i26.10831 - [Págs. 61-80]

El derecho a la educación y la seguridad en tiempos de Covid-19: Factores claves para la adopción de modelos de blended learning.. Amaya Gil Albarova / Cristina Monge Lasierra / Amparo Gracia Bernal / Federico Buyolo García

\section{KEYWORDS}

Educational inequality; improvement strategies; Covid-19; blended learning; face to face-virtual; techpedagogical mediation.

\section{SUMARIO}

1. INTRODUCCIÓN: EL SHOCK EDUCATIVO EN ESPAÑA DURANTE LA CRISIS DE LA COVID-19. 1.1. PRINCIPALES DATOS DE LA BRECHA DE USO. 1.2. PRINCIPALES DATOS DE LA BRECHA EDUCATIVA ESCOLAR. 2. METODOLOGÍA. 3. RESULTADOS: BLENDED LEARNING: UNA PROPUESTA PARA LA CONCILIACIÓN ENTRE LAACTIVIDAD EDUCATIVA Y LA SEGURIDAD SANITARIA. 3.1. QUÉ ES EL BLENDED LEARNING. 3.2. MODELOS DE APRENDIZAJE COMBINADO. 3.3. ANÁLISIS DE RESULTADOS POR MODELOS DEL BLENDED LEARNING UNIVERSE (B.L.U.). 3.4. PROCESO DE DISEÑO DE PROGRAMAS BLENDED LEARNING. 4. DISCUSIÓN: IDENTIFICACIÓN DE LOS FACTORES CRÍTICOS PARA LAADAPTACIÓN DE MODELOS DE BLENDED LEARNING EN ESCUELAS ESPAÑOLAS EN SITUACIÓN DE PANDEMIA. 4.1. FACTORES TRANSVERSALES. 4.2. FACTORES CRÍTICOS EN SITUACIÓN PANDEMIA. 5. CONCLUSIONES.

\section{INTRODUCCIÓN: EL SHOCK EDUCATIVO EN ESPAÑA DURANTE LA CRISIS DE LA COVID-19}

El desarrollo del curso 2019-2020 en España se ha visto bruscamente alterado por efecto de la Covid-19. Tras la suspensión de la actividad lectiva presencial, más de 9,5 millones de estudiantes se han visto afectados:

TABLA 1. TOtAL DE ALUMNADo MATRICULAdo EN EL CURSO 2019-2020

\begin{tabular}{|c|c|c|c|}
\hline & Total & $\begin{array}{l}\text { Centros } \\
\text { Públicos }\end{array}$ & $\begin{array}{l}\text { Centros } \\
\text { Privados }\end{array}$ \\
\hline TODAS LAS ENSEÑANZAS & 9.528 .261 & 6.749 .365 & 2.778 .896 \\
\hline TODAS LAS ENSEÑANZAS DE RÉGIMEN GENERAL & 8.217 .330 & 5.515 .197 & 2.702 .133 \\
\hline E. Infantil-Primer ciclo (1) & 470.461 & 239.816 & 230.645 \\
\hline E. Infantil-Segundo ciclo & 1.276 .913 & 860.317 & 416.596 \\
\hline E. Primaria & 2.938 .425 & 1.990 .188 & 948.237 \\
\hline Educación Especial & 37.302 & 22.516 & 14.786 \\
\hline ESO & 1.975 .358 & 1.299 .305 & 676.053 \\
\hline Bachillerato presencial & 631.779 & 458.356 & 173.423 \\
\hline Bachillerato a distancia & 35.647 & 34.279 & 1.368 \\
\hline Ciclos Formativos de FP Básica presencial & 74.009 & 55.963 & 18.046 \\
\hline Ciclos Formativos de FP Grado Medio presencial & 323.262 & 233.338 & 89.924 \\
\hline Ciclos Formativos de FP Grado Medio a distancia & 27.558 & 19.445 & 8.113 \\
\hline Ciclos Formativos de FP Grado Superior presencial & 353.821 & 255.001 & 98.820 \\
\hline Ciclos Formativos de FP Grado Superior a distancia & 60.114 & 37.746 & 22.368 \\
\hline Otros Programas Formativos & 12.681 & 8.927 & 3.754 \\
\hline ENSEÑANZAS DE RÉGIMEN ESPECIAL & 798.620 & 727.284 & 71.336 \\
\hline EDUCACIÓN DE PERSONAS ADULTAS & 512.311 & 506.884 & 5.427 \\
\hline
\end{tabular}

Fuente: Avance matriculación curso 2018-2019 MEFP. 
El Real Decreto 463/2020, de 14 de marzo, por el que se declara el estado de alarma para la gestión de la situación de crisis sanitaria ocasionada por la Covid-19 establece en su artículo 9 sobre medidas de contención en el ámbito educativo y de la formación:

«1. Se suspende la actividad educativa presencial en todos los centros y etapas, ciclos, grados, cursos y niveles de enseñanza contemplados en el artículo 3 de la Ley Orgánica 2/2006, de 3 de mayo, de Educación, incluida la enseñanza universitaria, así como cualesquiera otras actividades educativas o de formación impartidas en otros centros públicos o privados.

2. Durante el período de suspensión se mantendrán las actividades educativas a través de las modalidades a distancia y "on line", siempre que resulte posible».

Esta medida, tomada por las autoridades sanitarias con el fin de minimizar el impacto del contagio, ha llevado a la necesidad de tener que reconfigurar de forma improvisada y sin apenas tiempo de reacción un modelo educativo, haciendo que una educación concebida como netamente presencial y analógica, pase a desarrollarse, sin transición posible, con herramientas de educación a distancia on line. Este cambio radical, acrecentado además por la falta de recursos tanto materiales como pedagógicos, ha generado grave estrés tanto a las familias, como a los docentes y al alumnado.

Las desigualdades ya existentes, la falta de recursos, junto con el cierre de los centros educativos, configuran una situación de grave riesgo. UNESCO ${ }^{1}$ ha determinado las razones que explican por qué el cierre de los centros escolares puede ser perjudicial para el desarrollo formativo de los niños y niñas:

1. Interrupción del aprendizaje.

2. Alimentación.

3. Falta de preparación de los padres para la enseñanza a distancia o desde la casa.

4. Acceso desigual a las plataformas de aprendizaje digital.

5. Insuficiencias en materia de cuidado de los niños.

6. Costos económicos elevados.

7. Incidencia mecánica en el sistema de salud.

8. Aumento de la presión para los centros escolares que permanecen abiertos.

9. Tendencia al incremento de las tasas de abandono escolar.

Por su parte el Banco Mundial $(2020)^{2}$ alerta de las consecuencias que puede llevar aparejadas el cierre de los centros educativos: desde la pérdida en los aprendizajes, hasta el aumento de las tasas de abandono y la situación que viven muchos escolares que pierden la comida más importante del día. En el caso de España, esta realidad se ve agravada por las brechas educativas y sociales pre-existentes:

1. El índice $\mathrm{AROPE}^{3}$ en 2018 situaba al $28,8 \%$ de la población menor de 16 años en riesgo de exclusión social.

2. La tasa de abandono escolar temprano en 2019 se sitúa en el 17,3\% de los estudiantes en España. (MEFP, 2020).

3. La tasa de idoneidad del alumnado (INCE, 2000) se reduce en 17 puntos entre los 12 años $(86,5 \%)$ $y$ los 15 años $(69,5 \%)$.

Por otro lado, hay que destacar dos elementos que sitúan a España en una mala situación de partida para afrontar esta situación: la formación del profesorado y la innovación educativa.

Los índices de inversión educativa en España han ido cayendo a lo largo de los últimos años, encontrándonos en este momento por debajo de los países más avanzados y de aquellos con mejores resultados en las pruebas internacionales. Esta desinversión educativa se deja sentir de forma notable tanto en la formación del profesorado, como en investigación educativa. Ambos aspectos dificultan poder afrontar desde la previsión y la innovación los retos de la digitalización educativa.

En lo referente a la formación del profesorado vemos cómo desde el año 2009 las cifras han ido bajando de manera exponencial con un ligero repunte en 2015, momento en el que volvió a descender en la misma proporción al año siguiente. Hay que señalar que actualmente en España la inversión en formación del profesorado está por debajo de lo que se dedicaba en 1997.

\footnotetext{
https://es.unesco.org/covid19/educationresponse/consecuencias

https://blogs.worldbank.org/es/education/educational-challenges-and-opportunities-covid-19-pandemic

https://www.ine.es/jaxiT3/Tabla.htm?t=11201
} 
FiguRA 1. Evolución de LA INVERSIÓn EN EUROS FORMACIÓn DEL PROFESORAdo En ESPAÑA 1992-2018

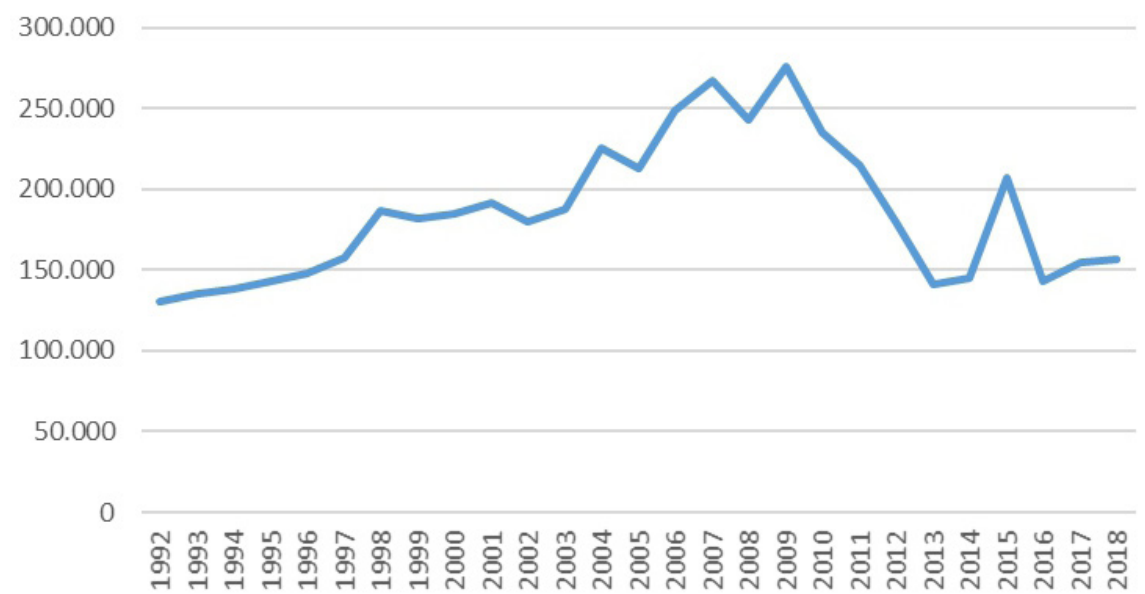

Fuente: Elaboración propia a través de los datos del MEFP (2020).

En lo concerniente a la inversión en investigación educativa los datos son igual de alarmantes, ya que las cifras de inversión se sitúan en los niveles de 2005, en torno a 48 millones de euros en 2018. Significativa es la curva descendente desde 2009, cuando se alcanzó la cifra máxima de 151 millones de euros, es decir, el triple de la cantidad que hoy se destina a la misma actuación en un mundo más digitalizado.

FiguRA 2. EVOLUCIÓN DE LA INVERSIÓN EN EUROS INVESTIGACIÓN EDUCATIVA EN ESPAÑA 1992-2018

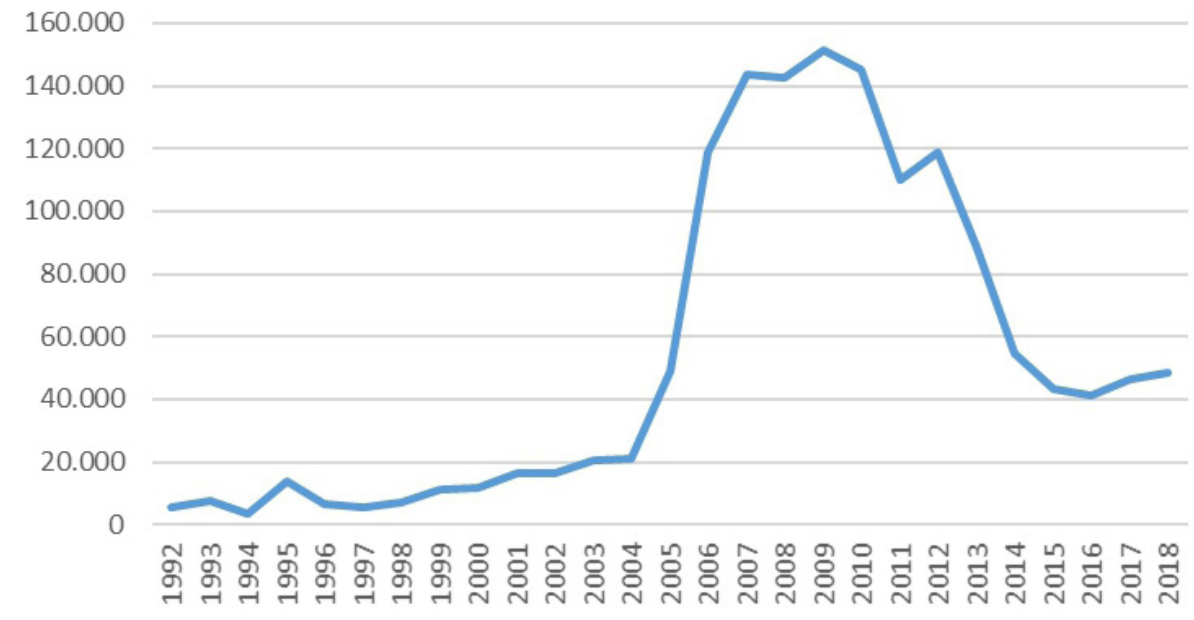

Fuente: Elaboración propia a través de los datos del MEFP (2020).

A estas circunstancias hemos de unirle la triple brecha digital, que hoy es más patente tras el cierre de los centros educativos. Al hablar de triple brecha digital ${ }^{4}$ se hace referencia a tres elementos:

1. Brecha de acceso: determinada como disponer o no de una conexión a internet y de dispositivos tecnológicos.

2. Brecha de uso: entendida como tiempo de uso de las tecnologías de la información y calidad del mismo para el estudio y aprendizaje.

3. Brecha educativa: medida por las habilidades del profesorado, la disponibilidad de recursos y adecuación de plataformas on line de apoyo a la enseñanza.

4 https://blog.enguita.info/2017/02/la-brecha-digital-es-ya-una-brecha.html 
Estos datos muestran algunas de las características que definen la brecha de acceso en España:

De los 3.142.214 hogares en España que no disponen de ordenador ni tablet, 792.000 son hogares donde viven niños y jóvenes en edad escolar y que carecen de medios para desarrollar un aprendizaje a distancia en condiciones óptimas (INE, 2019).

Otra de la circunstancia que agrava esta situación de falta de dispositivos en los hogares tiene que ver con la capacidad económica de las familias. La diferencia entre los hogares con mayores ingresos y los de menores ingresos (por debajo de 900 euros) es de 39,3 puntos (INE, 2019).

De los 1.408.630 hogares que no disponen de acceso a internet, 284.244 son hogares con hijos en edad escolar (INE, 2019), si bien hay que tener en cuenta que en muchos casos la conexión se hace mediante terminales de telefonía móvil.

Esta situación se ve agravada por la brecha económica en España. Solo el 77,9\% de los hogares con ingresos de menos de 900 euros dispongan de acceso a internet (INE, 2019). De los datos se deduce que el gap de acceso a internet entre las familias con mayores y menores ingresos es de 21,3 puntos.

\subsection{Principales datos de la brecha de uso}

En este momento de confinamiento se hace notar más la diferencia entre el uso de internet que se hace en el centro escolar y el que se realiza en el hogar, y se constatan diferencias muy significativas por comunidades autónomas. Mientras el uso en los centros educativos es muy alto en todas las comunidades, la diferencia entre el uso en el hogar es muy significativo, lo que puede convertirse en un factor de desigualdad importante.

Figura 3. Diferencias POR CC.AA del uso de internet EN CENTROS EDUCATIVOS Y EN EL HOGAR EN PORCENTAJE

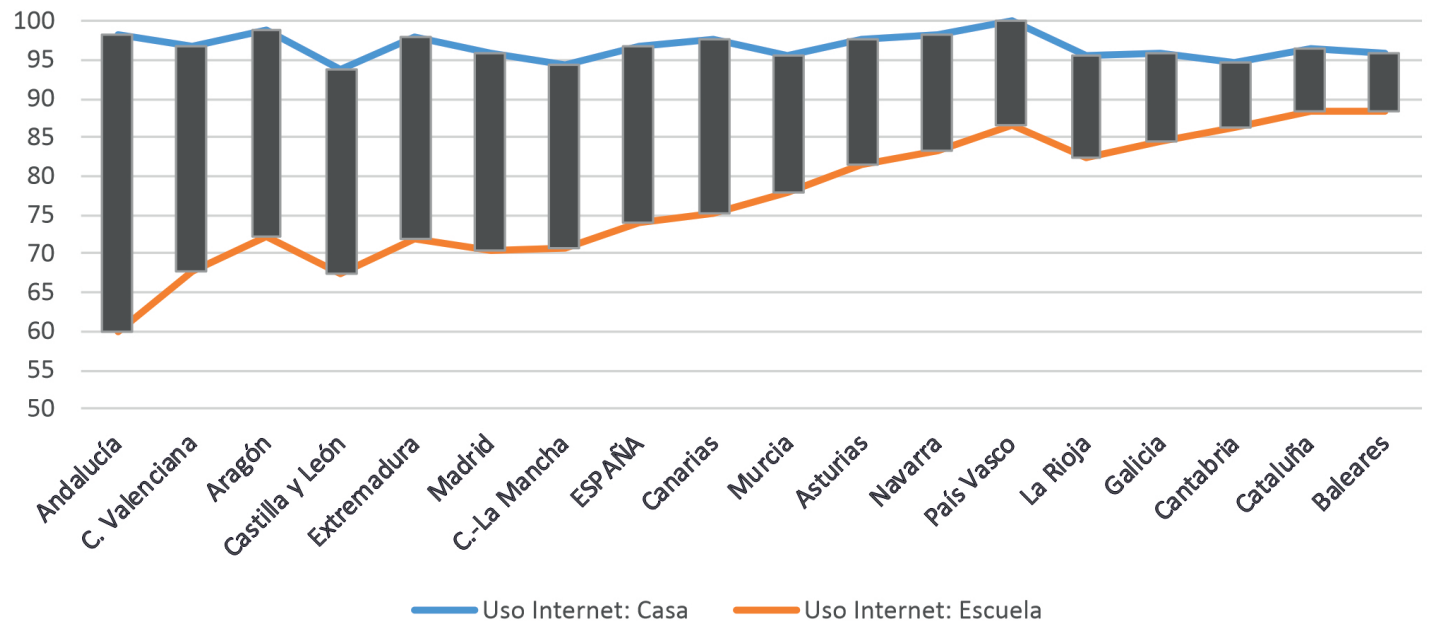

Fuente: Martínez Celorrio, X. (2019), a partir de encuesta sobre Equipamiento y uso de TIC, INE.

En los últimos tres meses de 2019 el número de niños que usaron internet fue superior al de acceso de los usuarios de ordenador, fundamentalmente debido a la extensión del uso del móvil entre los más jóvenes. En comparación con los países del entorno de la UE y la OCDE, el tiempo de uso de internet en los hogares españoles es similar a los hogares de la UE y menor que en la OCDE. En lo que se refiere al uso de internet por parte de la población para fines educativos hay que destacar que el nivel es muy bajo, ya que tan solo el $26,9 \%$ de las mujeres y el $26,3 \%$ de los hombres lo utilizaron como material de aprendizaje on line.

\subsection{Principales datos de la brecha educativa escolar (Eduteka, 2020)}

Al igual que hemos visto en los datos sobre el uso de internet por parte de los alumnos en los centros educativos, encontramos que la digitalización de la educación no está del todo interiorizada y desplegada en el sistema educativo español. Si hacemos un análisis de los principales elementos que necesita un centro educativo para la utilización de las tecnologías de la información aplicadas a la educación podemos comprobar cómo su implantación no se ha llevado a cabo de manera generalizada. 
GAPP. Nueva Época - N. ${ }^{\circ} 26$, julio 2021 - ISSN: 1989-8991 - DOI: https://doi.org/10.24965/gapp.i26.10831 - [Págs. 61-80]

El derecho a la educación y la seguridad en tiempos de Covid-19: Factores claves para la adopción de modelos de blended learning... Amaya Gil Albarova / Cristina Monge Lasierra / Amparo Gracia Bernal / Federico Buyolo García

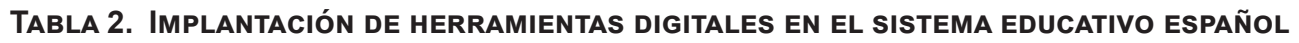

\begin{tabular}{lccccc}
\hline & Total & Centros & $\begin{array}{c}\text { Centros } \\
\text { públicos } \\
\text { Primaria }\end{array}$ & $\begin{array}{c}\text { Centros } \\
\text { Públicos } \\
\text { Secundaria }\end{array}$ & $\begin{array}{c}\text { Centros } \\
\text { Privados }\end{array}$ \\
\hline Página WEB & 86,5 & 84,2 & 80 & 95,1 & 94 \\
\hline Entornos Virtuales de aprendizaje & 40,1 & 37,3 & 25,4 & 68,6 & 49,1 \\
\hline $\begin{array}{l}\text { Participa en proyectos relacionados } \\
\text { con tecnología de la educación }\end{array}$ & 31,5 & 30,3 & 25,5 & 43 & 35,2 \\
\hline Sistemas digitales para impartir docencia & 66,5 & 64,2 & 70,0 & 55,7 & 73,2 \\
\hline
\end{tabular}

Fuente: Sociedad de la Información y la comunicación en los centros educativos 2016-2017 (MEFP).

Aunque los datos demuestran que el acceso a internet por parte de los alumnos gracias a los teléfonos móviles es más elevado, el porcentaje de centros que permiten la utilización del móvil con fines educativos es muy bajo, como se refleja en la siguiente tabla.

TABla 3. Centros que permiten el uso del móvil con fines educativos

\begin{tabular}{ccccc}
\hline ESO & BACHILLERATO & FP BÁSICA & FP GRADO MEDIO & $\begin{array}{l}\text { FP GRADO } \\
\text { SUPERIOR }\end{array}$ \\
\hline 34,5 & 41,5 & 30,2 & 41,0 & 45,5 \\
\hline
\end{tabular}

Fuente: Sociedad de la Información y la comunicación en los centros educativos 2016-2017 MEFP.

Por otro lado, hay que resaltar que la preparación de los docentes, tanto por recursos como por habilidades digitales, para afrontar esta situación, es especialmente acusada al compararlos por titularidad del centro:

\section{FIgURA 4. RECURSOS Y HABILIDADES EN EL MANEJO DE HERRAMIENTAS DIGITALES SEGÚN LA TITULARIDAD DE LOS CENTROS EDUCATIVOS}

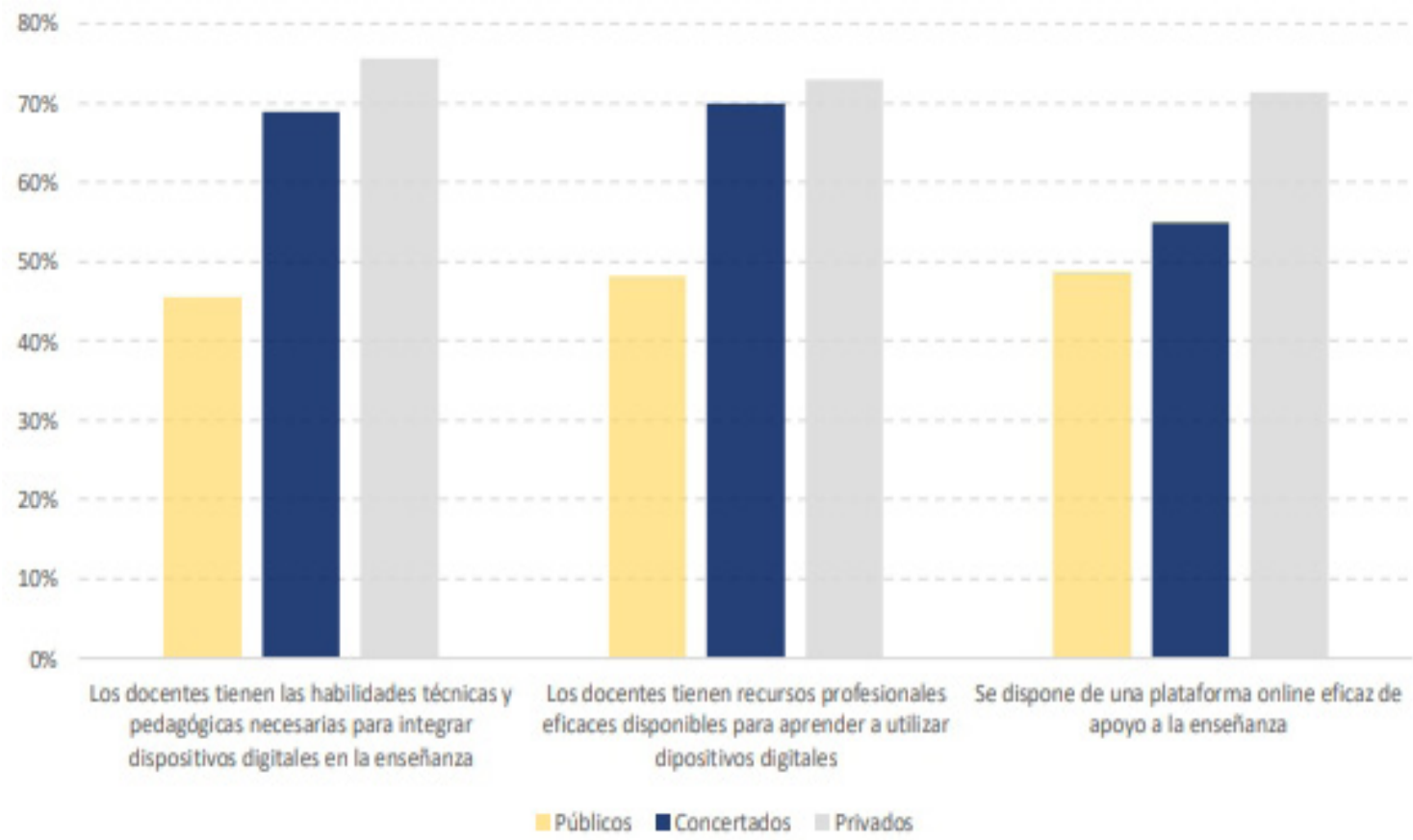

Fuente: COTEC a partir de los micro-datos de PISA 2018. 
Los datos demuestran que para lograr una implementación efectiva de modelos de blended learning es necesario afrontar inicialmente estas tres brechas digitales, además de la brecha económica y territorial que permita garantizar la igualdad de oportunidades de todos los alumnos y alumnas.

\section{METODOLOGÍA}

Para la elaboración de esta investigación se ha empezado por hacer una caracterización de la situación vivida en España a raíz de la Covid-19 revisando aquellas fuentes oficiales que aportaban datos de interés. A continuación, la descripción de los diferentes modelos de blended learning existentes se ha realizado tras revisar literatura especializada y comparada. Para la discusión se ha partido de investigaciones previas cuyas conclusiones se han aplicado a los modelos descritos. Finalmente se recogen los factores críticos para aplicar metodologías de blended learning en España una vez analizado el contexto y experiencias previas de este tipo de modelo educativo.

\section{RESULTADOS: BLENDED LEARNING: UNA PROPUESTA PARA LA CONCILIACIÓN ENTRE LA ACTIVIDAD EDUCATIVA Y LA SEGURIDAD SANITARIA}

El cierre de los centros educativos habría frenado el progreso de aprendizaje del alumnado (OEI, 2020). Más aún, de aquéllos que viven en entornos socioeconómicos más desfavorecidos (UNESCO, 2016). La falta de equidad ocasionada por la brecha digital puede estar siendo causa de mayor brecha educativa. "Abordar las desigualdades sociales y educativas fortalecerá nuestras sociedades y nos preparará para otros desafíos que podamos enfrentar" (Noorani y Croiser, 2020).

Según algunos análisis, este modelo de educación completamente on line, implantado de forma abrupta cuando se declaró la pandemia, no logra sustituir de forma eficaz la educación presencial sobre todo en determinadas etapas educativas (Fundación COTEC, 2020a y 2020b). De ahí que se estén buscando alternativas más favorables para mantener un equilibrio difícil de conseguir entre la actividad educativa y la seguridad sanitaria. Para abordarlas, conviene tener en cuenta algunos de los trabajos que vienen desarrollándose en el ámbito europeo:

- El Marco Europeo para Organizaciones Educativas Digitalmente Competentes (DigCompOrg) puede contribuir en la elaboración de políticas para la integración y uso eficaz de las tecnologías de aprendizaje digital, así como servir de guía para la autoevaluación de los centros educativos a medida que vayan avanzando en el desarrollo de pedagogías digitales (Kampylis, Punie, y Devine, 2015).

- Por su parte el Marco Europeo para la Competencia Digital de los Educadores (DigCompEdu), sirve como referencia para los gobiernos, los centros educativos, los proveedores de formación y los propios educadores para avanzar en las competencias digitales específicas para el docente de todas las etapas educativas (Redecker y Punie, 2017).

- También cobra interés el OpenEdu framework desarrollado para universidades, porque puede servir para tomar decisiones estratégicas sobre el uso de la educación abierta (R.E.A., MOOCs) que actualmente tiene un potencial enorme para desarrollar la innovación y facilitar el conocimiento disponible para todos (Inamorato dos Santos et al., 2016).

- A su vez se están desarrollando nuevos estudios para aumentar el conocimiento sobre cómo implementar las políticas de educación digital en la UE (DigEduPol) y comprender mejor los beneficios, los factores claves de éxito y las barreras todavía existentes (Conrads et al., 2017).

La urgencia de abordar el problema nos lleva a plantear propuestas que necesariamente concilien dos necesidades ineludibles: la seguridad sanitaria y la actividad educativa. En la búsqueda de alternativas, la enseñanza mixta (presencial y on line) se plantea como una solución que podría ser adecuada para el cambio temporal o definitivo de la forma de enseñar y aprender.

\subsection{Qué es el blended learning}

Existen varias definiciones de blended learning o hybrid learning para referirse a la modalidad de enseñanza que combina deliberadamente el aprendizaje en línea con la enseñanza presencial (Bartolomé, 2004). El aprendizaje combinado se concibe como un diseño pedagógico que mezcla las mejores prácticas de la 
enseñanza presencial (face-to-face) con las de la enseñanza en línea. Los rasgos que lo caracterizan y por los que puede ser un enfoque a tener en cuenta son la flexibilidad, la mayor eficiencia de los recursos (Bartolomé, 2004) y especialmente la mejora del rendimiento de los alumnos (Kenney y Newcombe, 2011; Stein y Graham, 2013). También por ser considerado un modelo más centrado en el alumno, más personalizado, que permite obtener mejores resultados con el mismo coste (Staker y Horn, 2012).

Esa eficiencia hace que sea una tendencia que se ha consolidado porque ha impactado realmente en el aprendizaje (Alammary et al., 2014; Alexander et al., 2019); además los alumnos muestran una preferencia por el aprendizaje combinado gracias a su flexibilidad y la integración de los elementos multimedia (Alexander et al., 2019).

Para el Blended Learning Universe -en lo sucesivo B.L.U.-, un centro de innovación en línea comisariado por el Clayton Christensen Institute dedicado a la innovación, el aprendizaje combinado es un programa de educación formal en el que el alumnado aprende en línea con algún sistema de control, y presencialmente en un aula supervisada por docentes. Ambas modalidades están conectadas a lo largo de una ruta de aprendizaje para proporcionar una experiencia integrada.

Según el B.L.U. el aprendizaje combinado tiene el potencial para transformar la educación definitivamente en tres dimensiones: impulsa el aprendizaje personalizado gracias a los datos individuales que proporciona y a la flexibilidad; desarrolla el aprendizaje basado en competencias, ya que los alumnos avanzan a su propio ritmo y no están condicionados por el tiempo; y se produce el aprendizaje en cualquier lugar y en cualquier momento, gracias a la tecnología que permite a los estudiantes llegar más allá de lo que ven en el aula.

Podría decirse que es un modelo de éxito a juzgar por el incremento de esta modalidad en centros de educación en todo el mundo (García-Ruiz et al., 2018) y por los buenos resultados tanto en la mejora del aprendizaje como en la satisfacción de los alumnos y de los docentes (Boyle et al., 2003; Hinojo y Fernández, 2012; Mackey y Watson, 2015; Siemens et al., 2015; Stein y Graham, 2013).

Según el MOOC titulado "BlendedX: Blended learning with EDX" de los profesores Jorge, Watson y Morris, el aprendizaje combinado tiene beneficios importantes para los estudiantes y los docentes:

- Al estar más centrado en el aprendizaje, se desarrollan habilidades metacognitivas de los estudiantes que consiguen aplicar estrategias de aprendizaje más personalizadas. Así logran aumentar notablemente la capacidad de aprender de forma autónoma y autodirigida, al propio ritmo y con mejores resultados.

- Permite una mayor flexibilidad a los docentes y a los estudiantes. Se logran los mismos o mejores resultados de aprendizaje con menos tiempo en clase, lo que permite que puedan completar su formación con otros intereses. Además, debido a que los docentes facilitan el contenido en línea, éstos también tienen la oportunidad de utilizar mejor el tiempo de la clase para innovar y probar otros formatos diferentes a las clases magistrales.

- Tanto los docentes como el alumnado pueden obtener información inmediata sobre el avance del aprendizaje. Los docentes pueden usar una variedad de instrumentos para recopilar información y realizar evaluaciones formativas. Los estudiantes pueden monitorizar su propio progreso y valorar en qué necesitan dedicar más tiempo, y en su caso pedir ayuda a profesores y compañeros.

- Permite crear oportunidades para mejorar el acceso al aprendizaje de nuevos colectivos de estudiantes no tradicionales y estudiantes con discapacidades físicas u otras dificultades de aprendizaje.

- Permite mejorar la alfabetización digital de alumnos y docentes (Alammary et al., 2014). A medida que se vuelven más expertos en el uso de la tecnología, hay mayores expectativas de que la utilicen con fines de enseñanza y aprendizaje.

Los docentes con experiencia en la impartición de cursos de aprendizaje combinado afirman convencidos que los revitaliza como docentes y que disfrutan experimentando con estructuras innovadoras y diseñando actividades de aula más atractivas, como se indica en el MOOC antes citado.

Por otro lado, el B.L.U. Directory Data 2016-2019 es una base de datos informativa sobre el aprendizaje combinado publicada en noviembre de 2019 como resultado de su investigación. Permite explorar datos de centros educativos de todo el mundo que han implantado programas de aprendizaje combinado. Incluye información sobre los modelos, tecnologías utilizadas y valoraciones sobre los resultados obtenidos. De un total de 538 centros de siete países de todo el mundo, 244 corresponden a EE.UU, por lo que es el país que presenta un mayor número de centros con datos disponibles sobre programas de aprendizaje combinado en las diferentes etapas educativas, durante los años 2004 a 2015. En la Tabla 4 se presenta la distribución según la ubicación de esos centros. 
GAPP. Nueva Época - N. ${ }^{\circ} 26$, julio 2021 - ISSN: 1989-8991 - DOI: https://doi.org/10.24965/gapp.i26.10831 - [Págs. 61-80]

El derecho a la educación y la seguridad en tiempos de Covid-19: Factores claves para la adopción de modelos de blended learning... Amaya Gil Albarova / Cristina Monge Lasierra / Amparo Gracia Bernal / Federico Buyolo García

Tabla 4. Centros de Estados Unidos que han implementado modelos de blended learning

\begin{tabular}{lc}
\hline \multicolumn{1}{c}{ Centros de EE.UU } & Núm. de centros \\
\hline Centros rurales & 21 \\
Centros suburbanos & 87 \\
Centros urbanos & 136 \\
\hline
\end{tabular}

Fuente: Elaboración propia a partir de B.L.U. Directory Data 2016-2019.

De esta selección de centros de los EEUU a continuación se cuantifican los centros que indican que las experiencias de aprendizaje combinado realizadas sirven para cada etapa y curso del sistema educativo norteamericano. Para conocer las equivalencias entre etapas y cursos, en la Tabla 5 puede observarse una mínima comparativa entre los sistemas educativos estadounidenses y españoles.

TABLA 5. UTILIDAD DE PROGRAMAS DE APRENDIZAJE COMBINADO POR CURSO Y ETAPA Y COMPARATIVA ENTRE EL SISTEMA EDUCATIVO ESPAÑOL Y ESTADOUNIDENSE

\begin{tabular}{|c|c|c|c|c|}
\hline \multicolumn{2}{|c|}{ Equivalencia al Sistema educativo español } & \multicolumn{2}{|c|}{ Sistema educativo USA } & $\begin{array}{c}\text { Núm. de centros en } \\
\text { USA que indican la } \\
\text { utilidad del blended } \\
\text { learning }\end{array}$ \\
\hline \multirow[t]{2}{*}{ Educación infantil } & Hasta 3 años & \multirow[t]{2}{*}{ Pre-school } & Pre-K & 3 \\
\hline & De 3 a 6 años & & Kindergarten & 74 \\
\hline \multirow{6}{*}{$\begin{array}{l}\text { Educación primaria } \\
6 \text { a } 12 \text { años }\end{array}$} & $1 .^{\circ}$ curso & \multirow{5}{*}{$\begin{array}{l}\text { Elementary school } \\
\text { (grado 1-grado 5) }\end{array}$} & First grade & 84 \\
\hline & $2 .^{\circ}$ curso & & Second grade & 87 \\
\hline & $3 .^{\circ}$ curso & & Third grade & 95 \\
\hline & $4 .^{\circ}$ curso & & Fourth grade & 88 \\
\hline & $5 .^{\circ}$ curso & & Fifth grade & 91 \\
\hline & $6 .^{\circ}$ curso & \multirow{3}{*}{$\begin{array}{l}\text { Middle school } \\
\text { (grado 6-grado 8) }\end{array}$} & Sixth grade & 108 \\
\hline \multirow{4}{*}{$\begin{array}{l}\text { Educación Secundaria } \\
\text { Obligatoria (ESO) } \\
12 \text { a } 16 \text { años }\end{array}$} & $1 .^{\circ}$ de ESO & & Seventh grade & 99 \\
\hline & $2 .^{\circ}$ de ESO & & Eighth grade & 99 \\
\hline & $3 .^{\circ}$ de ESO & \multirow{4}{*}{$\begin{array}{l}\text { High school } \\
\text { (grado 9-grado 12) }\end{array}$} & Ninth grade & 127 \\
\hline & 4. ${ }^{\circ}$ de ESO & & Tenth grade & 128 \\
\hline \multirow{2}{*}{$\begin{array}{l}\text { Bachillerato } \\
16-18 \text { años }\end{array}$} & 1. ${ }^{\circ}$ Bachillerato & & Eleventh grade & 118 \\
\hline & 2. ${ }^{\circ}$ Bachillerato & & Twelfth grade & 115 \\
\hline
\end{tabular}

Fuente: Elaboración propia a partir de B.L.U. Directory Data 2016-2019.

A la vista de los resultados, la cuestión que se plantea es cómo aplicar el aprendizaje combinado para adaptarlo a cada una de las etapas educativas según sus características y peculiaridades. Por ello conviene hacer una revisión de los diferentes modelos de este tipo de aprendizaje para que sirvan de guía a los centros educativos.

\subsection{Modelos de aprendizaje combinado}

Staker y Horn (2012) describen cuatro modelos principales: flexible, a la carta, virtual enriquecido y rotación. De este último a su vez se derivan cuatro submodelos: rotación de estación, rotación de laboratorio, 
rotación individual y aula invertida (flipped classroom). Los modelos se pueden implementar de forma única o bien en combinación entre sí. Lo que tienen en común siempre es la mezcla del aprendizaje en línea con el aprendizaje presencial.

1. El modelo Flexible (Flex) es uno de los más utilizados y se fundamenta en el aprendizaje en línea a través de una plataforma (EVA) y en un mayor control del alumno sobre su aprendizaje. Los docentes brindan apoyo e instrucción de manera flexible según sea necesario, en grupos pequeños o tutorías individuales, mientras los estudiantes trabajan a su ritmo en el contenido del currículo a través de la plataforma. La flexibilidad del modelo es considerada un factor fundamental para avanzar en la enseñanza personalizada (Clayton Christensen Institute).

En la Conrad High School, Montana, un centro equivalente a la educación primaria, en 2017 los maestros diseñaron el plan de estudios en una plataforma LMS y establecieron un sistema de incentivos con fichas para llevar el seguimiento del progreso del aprendizaje. Los estudiantes trabajan a su propio ritmo en el aula, y deben alcanzar un mínimo de cuatro fichas por semana para mantener la competencia al $80 \%$ o más. Cada día comienzan revisando el horario con su docente asesor para asegurarse de que están manteniendo el progreso, hacer y responder preguntas y construir relaciones. Los maestros se comunican con los padres al menos cada dos semanas. Van perfeccionando el modelo año a año (B.L.U.).

2. El modelo a la carta, recibe este nombre porque los estudiantes deciden qué cursos on line seguir para completar su currículo, además de la actividad presencial. Es uno de los modelos más populares en las escuelas secundarias en las que a menudo no hay suficiente oferta de asignaturas optativas.

Pamoja es un centro que ofrece cursos en línea dirigido al Programa del Bachillerato Internacional (IB) para complementar al programa existente del centro educativo. En la Halcyon London International School, un centro pequeño de IB en el Reino Unido, los estudiantes siguen estos cursos con dispositivos personales en un espacio de aprendizaje abierto para hacer su trabajo de forma independiente. Están rodeados otros diez compañeros que trabajan en diferentes cursos y son supervisados por un docente que puede ayudar a resolver problemas tecnológicos y académicos (B.L.U.).

3. El modelo virtual enriquecido es una alternativa a la docencia en línea a tiempo completo. Permite a los estudiantes completar la mayoría del trabajo escolar en línea en sus hogares o fuera del centro educativo, pero deben asistir al centro educativo en los días designados para las sesiones de aprendizaje presenciales con el docente, que generalmente es el mismo para la docencia en línea y presencial. Las sesiones presenciales están destinadas a enriquecer la experiencia de aprendizaje con trabajos grupales y al seguimiento regular de los alumnos por parte de sus profesores y tutores. A diferencia del aula invertida, este modelo no requiere asistencia diaria a la escuela; algunos programas solo requieren asistencia dos veces por semana.

Aunque es más frecuente en la educación superior, está teniendo auge en Centros educativos de secundaria como la premiada Da Vinci RISE High School de California o la Map Academy de Plymouth que han aprovechado el modelo virtual enriquecido para cambiar la forma en que los estudiantes y los maestros usan el tiempo y el espacio para crear experiencias de aprendizaje dinámicas (B.L.U.).

4.1. El modelo Rotación de estación, en el que los estudiantes rotan en grupo dentro de una misma aula a través de diferentes «estaciones» en horarios fijos en lo que sería una ruta de aprendizaje. Necesariamente todos pasan por el emplazamiento de aprendizaje en línea, por el dedicado al trabajo colaborativo y la clase tradicional. En todos ellos los alumnos están supervisados por dos docentes (suele haber un segundo docente de apoyo).

El centro Mt. Gilead Middle Schoolen de Ohio, donde usaron el modelo Station Rotation, indicaron que les sirvió para conocer mejor a sus alumnos, evaluar y poder satisfacer mejor las necesidades individuales. La mejora del rendimiento de los alumnos les valió el Premio Momentum de Ohio de la escuela durante tres años seguidos (B.L.U.).

4.2. La Rotación de laboratorio es similar al anterior pero el aprendizaje en línea debe realizarse necesariamente en un laboratorio de computación acondicionado específicamente a ello con ordenadores, dispositivos, impresoras 3D, gafas de VR entre otras cosas. Este modelo permite la planificación conjunta y flexible entre docentes y otros profesionales de apoyo.

En el centro privado Guru Nanak National High School de Mumbai, el modelo Lab Rotation permite dividir a los alumnos en dos grupos. Un grupo está con el docente en el aula mientras el otro trabaja en un laboratorio informático con un asistente de enseñanza que facilita el aprendizaje. El programa de aprendizaje Zaya es adaptable al ritmo de cada alumno. Los grupos rotan entre estas dos «estaciones» en días alternos. Con los datos obtenidos de Zaya, se facilitan informes de aprendizaje personalizados para ayudar al estudiante y a ambos docentes para seguir el progreso de manera transparente (B.L.U.). 
4.3. La Rotación individual, aunque es un modelo parecido a los anteriores, el horario de cada estudiante es individual y está establecido por el docente o el algoritmo de una aplicación. A diferencia de los anteriores modelos de rotación, los estudiantes solo rotan por las actividades programadas en su ruta personalizada.

La Bennie Dover Jackson Middle School en Connecticut se asoció con la organización sin fines de lucro New Classrooms para reemplazar su plan de estudios de matemáticas por el modelo de aprendizaje personalizado Teach to One: Math. Los profesores y los estudiantes pueden seguir el aprendizaje a través de sus respectivos portales en línea. Los ciclos de aprendizaje duran aproximadamente tres semanas y en cada ronda, los estudiantes tienen la oportunidad de reunirse con un maestro y un grupo de compañeros varias veces para trabajar juntos en un proyecto. También incluyen un sistema de incentivos (B.L.U.).

4.4. El Aula invertida o flipped classroom (Bergmann y Sams, 2014) consiste básicamente en que el alumnado aprende nuevos conceptos en casa a través de cursos en línea mediante lecturas, videos y ejercicios, y el tiempo presencial en el aula se usa para las actividades prácticas interactivas o proyectos guiados y supervisados por el docente. Este enfoque ha ido madurando hasta llegar al modelo Micro-Flip Teaching (MFT) de metodologías activas en el que «las actividades en casa y las realizadas en clase forman un conjunto planificado de acciones. Unas no se pueden hacer sin las otras» (Fidalgo-Blanco et al., 2020). Los autores analizaron diferentes artículos científicos sobre la aplicación del aula invertida en diferentes contextos y presentan valiosos indicadores sobre la mejora del rendimiento académico de los alumnos.

\subsection{Análisis de resultados por modelos del Blended learning universe (B.L.U.)}

Del análisis de los resultados obtenidos por el B.L.U. de centros educativos en EE.UU que han implantado programas de aprendizaje combinado, se observa que los modelos sobre los que más datos se han obtenido son el modelo Rotación de estación y el modelo Flexible, por ese orden. Además, la utilización de los modelos descritos es diferente según el curso y el nivel educativo donde se está aplicando el aprendizaje combinado.

En los cursos equivalentes a la etapa de Educación Primaria, el modelo Rotación de estación es el más utilizado de todos con una evolución significativa en los últimos años (Fig. 5).

FiguRA 5. MOdELOS IMPLEMENTAdOS EN LAS ESCUELAS PRIMARIAS

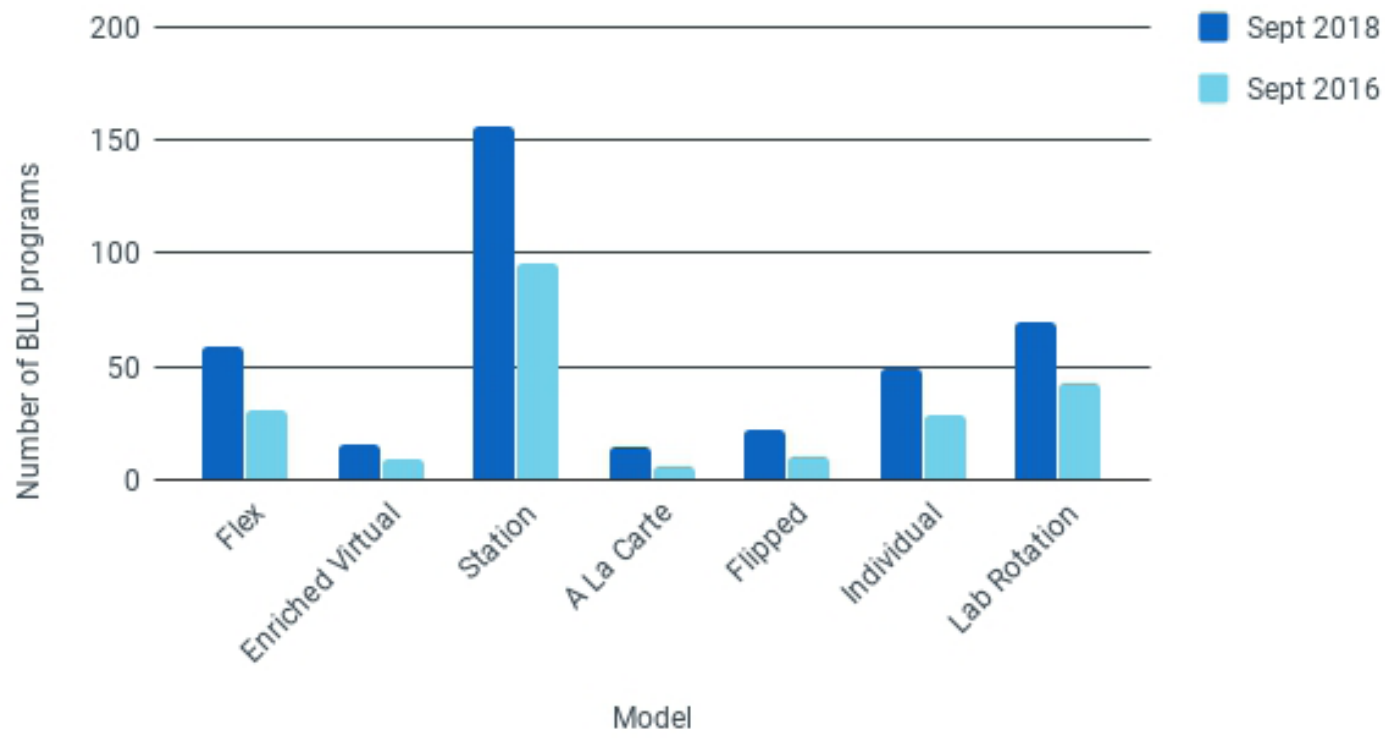

Fuente: Blended learning universe.

En los cursos equivalentes a $6 .^{\circ}$ Educación Primaria, $1 .^{\circ}$ y $2 .^{\circ} \mathrm{ESO}$, también destaca la popularidad del modelo Rotación de estación, aunque también se utilizan el Flexible y el resto de los modelos de rotación. Entre 
los testimonios de docentes recogidos, señalan que la Rotación individual permite atender mejor a los estudiantes con dificultades y permite al docente ganar más tiempo para la enseñanza en grupos pequeños (Fig. 6).

FiguRA 6. MOdelos implementados en enseñAnZAS MEdiaS

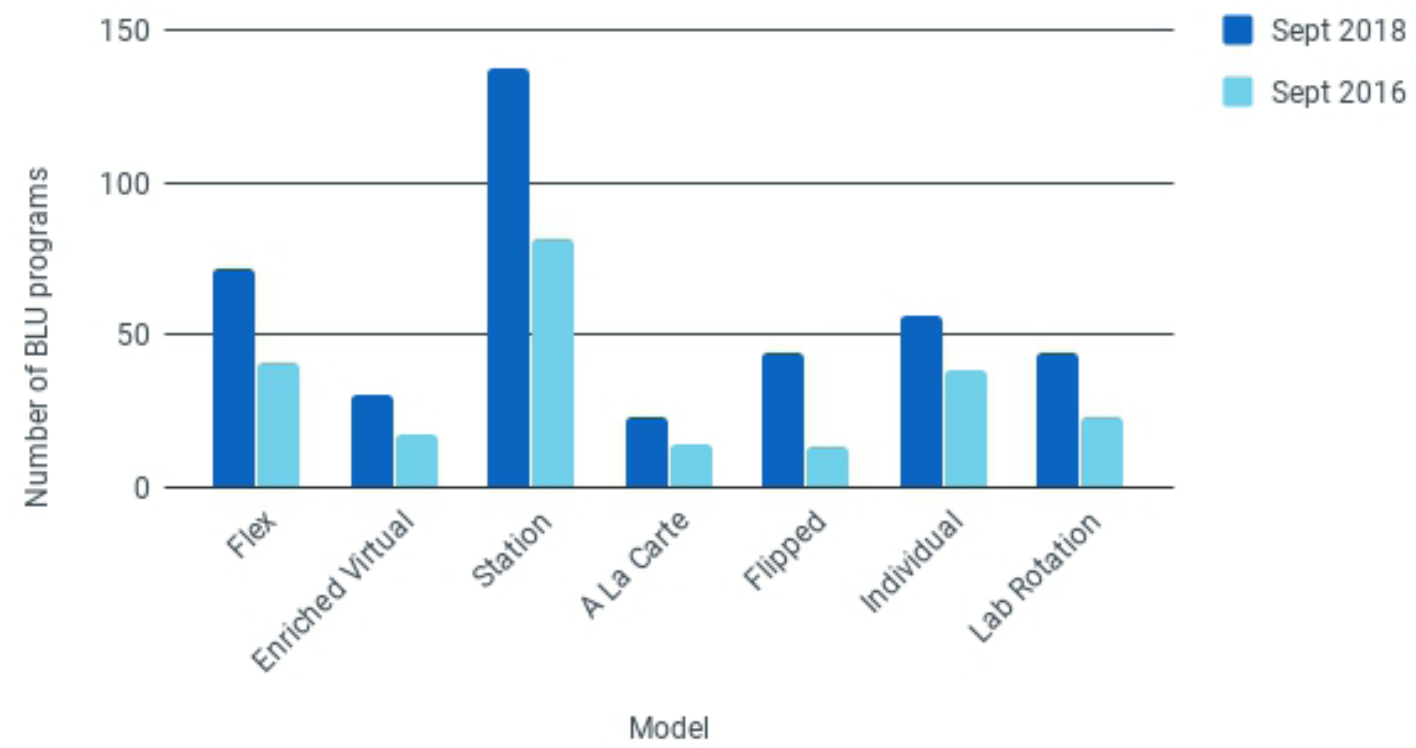

Fuente: Blended learning universe.

En los niveles más altos, los equivalentes a $3 .^{\circ}, 4 .^{\circ}$ de ESO y $1 .^{\circ}$ y $2 .^{\circ}$ Bachillerato, el modelo Flexible es el que ha sido más indicado porque permite a los alumnos un mayor control sobre su aprendizaje y la personalización de su horario, de modo que los acerca a una experiencia más parecida a la de la educación superior (Fig. 7). Un ejemplo de ello es la Red de escuelas estadounidense High Tech High de San Diego, que ha establecido un sistema de b-learning en el que muchas de las clases se dividen a la mitad entre formación presencial y formación digital, y con un enfoque de aprendizaje basado en proyectos, están obteniendo magníficos resultados en el acceso a universidad.

Figura 7. Modelos implementados en ENSEÑANZA SECUNDARIA

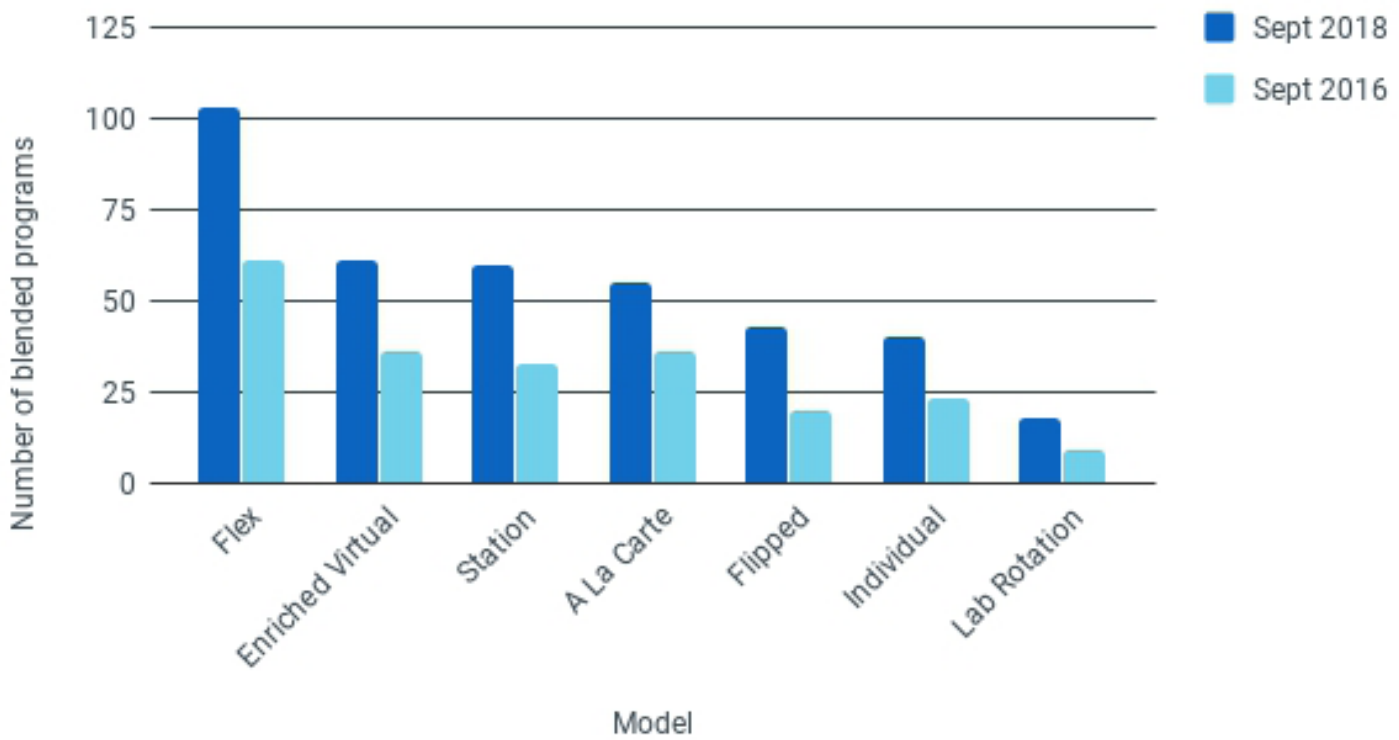

Fuente: Blended learning universe. 
A la vista de los datos del B.L.U. para todos los niveles de grado en 2016 y 2018 , se puede constatar que la tendencia a la adopción de modelos combinados va en aumento, y que los modelos más utilizados continúan siéndolo con el tiempo. Cuando se compara las tendencias entre Estados Unidos y otros países, se puede observar que las tendencias entre los modelos difieren (Fig. 8).

Figura 8. MOdeLos de APRENDIZAJE híBRIDO UTILIZADOS A NIVEL INTERNACIONAL

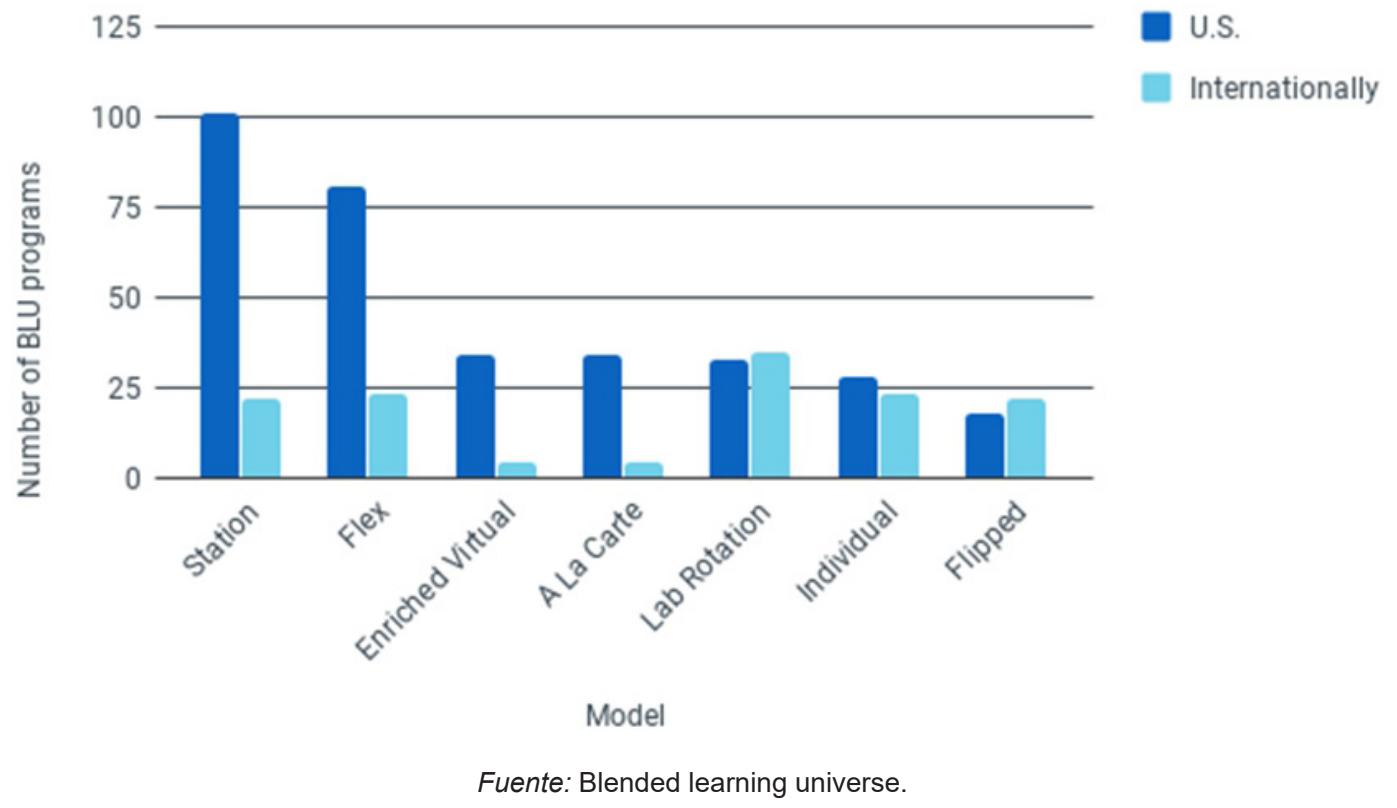

Para adaptarlo a cada centro y etapa deben considerarse las circunstancias del centro educativo y su contexto socioeconómico. Independientemente de ello, los buenos resultados siempre van a depender de tres factores claves de éxito: la cultura de innovación que exista en el centro educativo (Alammary et al., 2014), la disponibilidad de recursos, y de forma especial, la motivación y el compromiso del profesorado implicado (Staker, 2011).

\subsection{Proceso de diseño de programas Blended learning}

El proceso para el diseño de programas de aprendizaje combinado que se plantea en el B.L.U. fue diseñado por Heather Staker y Michael Horn y se compone de diez pasos. Cada uno de ellos incluye herramientas útiles para la toma de decisiones y la planificación de acciones:

1. Identificar el problema a resolver o la meta a alcanzar.

2. Formar un equipo de personas adecuadas para trabajar en el diseño, es la clave de éxito.

3. Diseñar el modelo pensando en el alumno y en cómo motivarlo.

4. Desarrollar todo el potencial de los docentes impulsando nuevos roles para dedicar tiempo a tareas valiosas como las tutorías o el diseño de experiencias de aprendizaje.

5. Decidir la estrategia tecnológica y proveedores según las prioridades del centro: calidad, flexibilidad, hiperpersonalización de la enseñanza.

6. Rediseñar las aulas como espacios de aprendizaje para mejorar la experiencia de los alumnos.

7. Seleccionar el modelo de aprendizaje combinado más adecuado para el centro.

8. Desarrollar deliberadamente una cultura de aprendizaje combinado en el equipo docente para resolver los problemas recurrentes.

9. Al tratarse de un proceso continuo, conviene preparar una lista de suposiciones sobre la organización del aprendizaje combinado y clasificarlos por orden de importancia.

10. Debe contar con un proceso de evaluación sistemática para monitorizar el impacto en el aprendizaje y la satisfacción de los alumnos, así como el impacto en la enseñanza y no menos importante, la capacidad de gestión inmediata de las incidencias (Garrison y Kanuka, 2004). 


\section{DISCUSIÓN: IDENTIFICACIÓN DE LOS FACTORES CRÍTICOS PARA LA ADAPTACIÓN DE MODELOS DE BLENDED LEARNING EN ESCUELAS ESPAÑOLAS EN SITUACIÓN DE PANDEMIA}

Es necesario establecer una serie de pautas que, con carácter transversal, la evidencia empírica ha demostrado son importantes para el éxito de los programas a desarrollar. Se trata de la vinculación de la educación con las políticas laborales, la flexibilidad del sistema, el uso que el alumnado hacía de las TIC antes de la pandemia, la relación inexcusable de las familias con el centro educativo y la formación del profesorado. Dado el objetivo del artículo, centrado en determinar los factores claves para la adopción de un modelo de blended learning, se ha optado por profundizar en los tres últimos factores, dejando los dos primeros para futuras investigaciones.

\subsection{Factores transversales}

1. Valoración del uso de las TIC por el alumnado en el escenario previo a la Covid-19.

Antes de la pandemia generada por la Covid-19 existían valoraciones diferentes respecto a los efectos del uso de las TIC por parte del alumnado. Por un lado, estaban aquellas que defendían que el impacto del uso de las TIC en la educación generaba un rendimiento en los alumnos muy escaso, basándose en los estudios del impacto de su uso, en la eventual reducción de las dificultades socioeducativas y en el rendimiento que resulta aún más escaso. Todo ello parece indicar un incremento de la brecha académica (Volman et al., 2005). Según esta valoración ¿podría el Covid-19 haber venido a incrementar la brecha académica o la mantendría estable?

Por otro lado, se podían encontrar autores que sostienen, al contrario, que el uso de las TICs por parte del alumnado mejora sustancialmente el rendimiento educativo del alumno (Harris y Smith, 2004; Lei y Zhao, 2008). En línea con esta segunda postura se encuentra el reciente estudio Tablet PCs, academic results and educational inequalities, realizado por la UOC en las escuelas de educación primaria de Aragón, respecto del uso de TIC, que concluyó que:

- El uso individual de las tabletas en las escuelas primarias contribuye a la reducción de las desigualdades socioeducativas.

- Los alumnos nacidos en países extranjeros se sienten más empoderados cuando usan tabletas PC que sus contrapartes nacidos en España.

- Los estudiantes con peores registros académicos encuentran que sus resultados mejoran más en comparación con sus compañeros de clase.

- Los estudiantes procedentes de entornos socioeconómicos más desfavorecidos son los que más se han beneficiado del uso de tabletas en educación primaria (Ferrer et al., 2010, p. 287).

Según se muestra, el uso de TICs puede ayudar a paliar algunas de las dimensiones de la desigualdad, por lo que su uso puede ser positivo en situaciones como las que se abordan.

\section{Situación de las familias y el obligado tándem con la escuela.}

Los principales estudios mundiales, Encuesta Social Europea, informe FOESSA, informe PISA, etc., refrendan que la realidad socioeducativa de los alumnos depende de las variables sociales fundamentales (género, lugar de nacimiento, padres, nivel de educación de la madre, del padre...).

La crisis de la Covid-19 ha generado una forma de convivencia en confinamiento en la que una buena parte de los progenitores ha teletrabajado y/o conciliado la vida familiar y laboral, lo que podría haber transformado los vínculos o las conexiones escuela-familia. En el estudio realizado en Madrid sobre estrategias para padres y dinámica de participación en una escuela de clase media, "Privileging the Individual Through the Collective Commitment: Parental Strategies and Dynamics of Involvement in a Middle-Class School" se concluye que las familias son actores educativos que se transforman durante el proceso de escolarización de sus hijos (González-Patiño y Poveda, 2015, p. 320). Para el caso español, defienden los autores, las relaciones entre la escuela, la familia y la comunidad todavía están en disputa y no han sido resueltas en parte por los cambios legislativos en torno a la educación, que no ha ayudado a favorecer un consenso sobre cómo los maestros y padres, las escuelas y las comunidades deben comunicarse e interactuar entre sí. 
Las continuidades familia-escuela se interpretan consistentemente como un factor que tiene un impacto en la educación y el desarrollo de los niños. En el caso español, implica construir, promover y limitar activamente versiones particulares de lo que constituye la participación de los padres y de ser un «padre de la escuela» (González-Patiño y Poveda, 2015, p. 321).

\section{Formación del profesorado.}

Como se señalaba más arriba, la formación del profesorado y la existencia de entornos y plataforma digitales adecuadas forma parte también de la brecha digital. La digitación de la educación ha de contemplar la mejora de las competencias digitales del profesorado. Esta formación debe asegurar no solo la utilización de las herramientas digitales, sino que ha de estar centrada en una adaptación de las formas de enseñanza, aprendizaje, evaluación y seguimiento del proceso educativo orientado a la mejora de los aprendizajes del alumnado. Un modelo educativo no migrado, sino construido sobre el nuevo entorno y las nuevas capacidades que ofrece el blended learning.

\subsection{Factores críticos en situación pandemia}

La pandemia por Covid-19 ha traído consigo la necesidad urgente de replantear el modelo educativo para mantener activa la actividad educativa a la vez que se aplican medidas de prevención sociosanitaria para preservar la salud de alumnos, docentes y personal de administración y servicios de los centros educativos.

Cuando se plantea la reapertura de los centros educativos, las cuestiones a resolver son definir los grupos de alumnos, los horarios, los turnos de estancias, las aulas y los espacios a utilizar, las normas de desplazamiento y las tareas que se pueden o no hacer en casa y las que necesariamente deben realizarse en el centro de forma presencial, entre otras cosas. En este sentido, se están planteando posibilidades como:

- Mantener el horario de clases y ampliar el número de aulas para distribuir a los alumnos en grupos más pequeños. El problema es que no suele haber número de aulas suficientes ni tampoco docentes para atender tantos pequeños grupos.

- Ampliar el horario de clases distribuyendo a los alumnos por franjas y así asegurar el mínimo número de estudiantes al mismo tiempo en las mismas aulas del centro. El problema es la limitación del profesorado y de sus jornadas.

- Plantear modelos de alternancia entre la docencia presencial y la docencia on line.

Si bien el enfoque de aprendizaje combinado no se ha aplicado nunca en una situación de pandemia como la que vivimos, algunos de los modelos descritos pueden aportar soluciones que permitan coordinar la necesidad de mantener activo la actividad educativa con las medidas de seguridad sanitaria preceptivas (uso de mascarillas, higiene y en especial el distanciamiento social).

Con el objetivo de ayudar a las autoridades educativas y los responsables de los centros educativos a tomar decisiones de organización de la docencia que garantice la seguridad sanitaria, y con la evidencia científica aquí reflejada, se analizan los modelos de aprendizaje combinado más adecuados al aplicar dos criterios claves comunes en todos los centros:

1. El distanciamiento físico como una de las medidas fundamentales que requiere limitar el aforo en los espacios y mantener al menos dos metros de distancia entre las personas. Por tanto, es necesario procurar el menor número de alumnos posibles en el aula o espacios educativos muy grandes.

2. Incrementar la eficiencia intentando abordar la solución con el mismo número de docentes y personal de apoyo, de aulas y equipos informáticos disponibles en el centro educativo.

Como vemos en cuanto a recursos necesarios, el modelo virtual enriquecido y el aula invertida son los más eficientes en tanto que sólo necesitan un profesor y un aula para atender al mismo número de alumnos, pero en ambos casos es necesario que todos los alumnos dispongan de dispositivos. A la misma conclusión llega Arnett (2020), investigador principal en educación del Christensen Institute cuando plantea el B-learning como posibilidad para reabrir las escuelas en otoño.

Sin embargo, desde el punto de vista pedagógico, a la hora de elegir el modelo más adecuado para etapa educativa según los datos disponibles del B.L.U., las prioridades cambian. A continuación se presenta el orden de preferencia de los modelos por niveles educativos, siendo los que llevan el número 1 y 2 los que tienen mayor popularidad. 
GAPP. Nueva Época - N. ${ }^{\circ} 26$, julio 2021 - ISSN: 1989-8991 - DOI: https://doi.org/10.24965/gapp.i26.10831 - [Págs. 61-80]

El derecho a la educación y la seguridad en tiempos de Covid-19: Factores claves para la adopción de modelos de blended learning...

Amaya Gil Albarova / Cristina Monge Lasierra / Amparo Gracia Bernal / Federico Buyolo García

Tabla 6. Necesidades de docentes, espacios y salas de ordenadores SEGÚN LOS MODELOS DE BLENDED LEARNING

\begin{tabular}{|c|c|c|c|c|c|c|c|}
\hline Necesidades & Flex & $\begin{array}{l}\text { A la } \\
\text { carta }\end{array}$ & $\begin{array}{c}\text { Virtual } \\
\text { enriquecido }\end{array}$ & $\begin{array}{l}\text { Rotación } \\
\text { estación }\end{array}$ & $\begin{array}{l}\text { Rotación } \\
\text { laboratorio }\end{array}$ & $\begin{array}{l}\text { Rotación } \\
\text { individual }\end{array}$ & $\begin{array}{c}\text { Aula } \\
\text { invertida } \\
\text { (Flipped } \\
\text { classroom) }\end{array}$ \\
\hline $\begin{array}{l}\text { Número mínimo } \\
\text { de docentes } \\
\text { necesarios. }\end{array}$ & 2 & $1-2$ & 1 & $1-2$ & 2 & 2 & 1 \\
\hline $\begin{array}{l}\text { Número aulas, } \\
\text { espacios de } \\
\text { aprendizaje, etc. }\end{array}$ & $5-7$ & $1-2$ & 1 & 1 & $2-4$ & $3-4$ & 1 \\
\hline $\begin{array}{l}\text { Aulas con } \\
\text { ordenadores } \\
\text { conectados para } \\
\text { la docencia en } \\
\text { línea. }\end{array}$ & Sí & Sí/No & No & Sí & Sí & Sí & No \\
\hline $\begin{array}{l}\text { Alumnos con } \\
\text { ordenadores } \\
\text { conectados desde } \\
\text { casa. }\end{array}$ & No & No & Sí & No & No & No & Sí \\
\hline
\end{tabular}

Fuente: Elaboración propia basado en BLU Directory Data 2016-2019.

Tabla 7. Preferencia de modelos b-Learning por etapa educativa

\begin{tabular}{lccccccc}
\hline & \multicolumn{7}{c}{ Orden de preferencia de modelo b-learning } \\
\cline { 2 - 8 } Nivel educativo & Flex & A la carta & $\begin{array}{c}\text { Virtual } \\
\text { enriquecido }\end{array}$ & $\begin{array}{c}\text { Rotación } \\
\text { estación }\end{array}$ & $\begin{array}{c}\text { Rotación } \\
\text { laboratorio }\end{array}$ & $\begin{array}{c}\text { Rotación } \\
\text { individual }\end{array}$ & $\begin{array}{c}\text { Aula } \\
\text { invertida }\end{array}$ \\
\hline Educación Infantil & 3 & 6 & 4 & 1 & 2 & 5 & 7 \\
\hline Educación Primaria & 3 & 7 & 6 & 1 & 2 & 5 & 5 \\
\hline $10^{\circ}$ y 2. ${ }^{\circ}$ ESO & 2 & 7 & 5 & 1 & 7 & 3 & 4 \\
\hline $3 .^{\circ}$ y 4. ${ }^{\circ}$ ESO & 1 & 4 & 2 & 3 & 7 & 6 & 5 \\
\hline Bachillerato y FP & 1 & 4 & 2 & 3 & 5 & 5 \\
\hline
\end{tabular}

Fuente: Elaboración propia basado en BLU Directory Data 2016-2019.

En los niveles de alumnos con menos edad, el modelo más utilizado es el de Rotación de estación, mientras que en los niveles de alumnos mayores son el modelo Flexible y Virtual enriquecido.

Por último, para identificar las tareas que necesariamente deben realizarse en el centro y las que pueden realizarse en casa, es una cuestión que va a depender fundamentalmente de la estrategia pedagógica y de la disponibilidad de entornos virtuales de aprendizaje, herramientas y recursos educativos abiertos a los que docentes y alumnos pueden acceder.

Si algo hemos aprendido durante las semanas de confinamiento es que todo puede ser posible si dedicamos tiempo a la formación de los docentes para probar las herramientas virtuales existentes para la docencia y el aprendizaje.

Entre las mejores herramientas en línea para aprender según la 13. ${ }^{a}$ Encuesta anual de herramientas de aprendizaje (Hart, 2019) observamos que muchas son abiertas o gratuitas como YouTube, paquetes de herramientas de Google y Microsoft para centros educativos, plataformas MOOCs, blogs de expertos, etc.; otras cuentan con versiones gratuitas para la creación de contenidos de aprendizaje (mapas mentales, tra- 
GAPP. Nueva Época - N. ${ }^{\circ} 26$, julio 2021 - ISSN: 1989-8991 - DOI: https://doi.org/10.24965/gapp.i26.10831 - [Págs. 61-80]

El derecho a la educación y la seguridad en tiempos de Covid-19: Factores claves para la adopción de modelos de blended learning... Amaya Gil Albarova / Cristina Monge Lasierra / Amparo Gracia Bernal / Federico Buyolo García

bajos en equipo, infografías...) y otras son software libre y pueden adaptarse a las circunstancias de cada centro como Moodle.

\section{CONCLUSIONES}

La «nueva normalidad» en la que discurrirá la vida en España tras el fin del confinamiento, es posible que reinvente la comunicación y la interacción, puesto que se han visto modificados condicionantes previos como son los horarios de trabajo y las complejidades espaciales de la vida urbana, que se han visto transformados en teletrabajo y confinamiento domiciliario. La evolución de la pandemia impide saber con exactitud cuál será la evolución de estos factores.

La pandemia ha provocado una transición abrupta a la educación a distancia on line que pone en evidencia las desigualdades existentes y la falta de recursos, pero también estaría acelerando forzosamente la adaptación digital de profesores y alumnos.

El enfoque de aprendizaje combinado no se ha aplicado nunca en una situación como la que vivimos, pero algunos de sus modelos pueden aportar soluciones que permitan conciliar la actividad educativa con las medidas de seguridad sanitaria preceptivas (el uso de mascarillas, la higiene de manos y en especial, la distancia interpersonal).

Los resultados obtenidos del análisis de la aplicación de estos modelos de aprendizaje combinado en centros de EE.UU antes de la pandemia, ponen de manifiesto que, en los niveles de alumnos con menos edad, el modelo de aprendizaje que valoran más adecuado desde el punto de vista pedagógico es el de Rotación de estación, mientras que en los niveles de alumnos mayores son el modelo Flexible y Virtual enriquecido.

En tiempos de Covid-19, al aplicar en el análisis los criterios de seguridad sanitaria y de disponibilidad de recursos en los centros, los modelos más eficientes serían el modelo virtual enriquecido y el aula invertida.

Entre los factores críticos para llevar a cabo la adaptación a modelos blended learning, cabría considerar la valoración del uso de las TIC por parte de autoridades educativas, alumnos, profesorado y familias, que serían más conscientes de la necesidad de formarse en competencias digitales. No sólo por la importancia de repensar los enfoques pedagógicos del proceso de enseñanza, aprendizaje y evaluación, sino también por la necesaria relación familia-escuela en la educación.

No obstante, la oportunidad de avanzar en soluciones educativas en tiempos de pandemia sólo será posible si logramos superar el mayor desafío, el de asegurar el acceso digital para todos, cuidando especialmente de los grupos más desfavorecidos para que no queden desconectados.

\section{REFERENCIAS BIBLIOGRAFICAS}

Alammary, A., Sheard, J. y Carbone, A. (2014). Blended Learning in higher education: Three different design approaches. Australian Journal of Educational Technology, 30(4), 440-454. https://doi.org/10.14742/ajet.693

Alexander, B., Ashford-Rowe, K., Barajas-Murph, N., Dobbin, G., Knott, J., McCormack, M., Pomerantz, J., Seilhamer, R. y Weber, N. (2019). 2019 Higher Education Edition [Horizon Report]. Educase. https://library.educause.edu/ resources/2019/4/2019-horizon-report

Arnett, T. (27 de mayo de 2020). The blended learning models that can help school reopen [entrada de blog]. Christensen Institute. https://www.christenseninstitute.org/blog/the-blended-learning-models-that-can-helpschools-reopen/

Bartolomé Piña, A. (2004). Blended Learning. Conceptos básicos. Pixel-Bit. Revista de Medios y Educación, 23, 7-20. https://recyt.fecyt.es/index.php/pixel/article/view/61237

Bergmann, J. y Sams, A. (2014). Dale la vuelta a tu clase. Ediciones SM.

Blended learning Universe (2019). BLU Directory Data [archivo de datos]. Christensen Institute. https://www. blendedlearning.org/wp-content/uploads/2019/11/Blended-Learning-Universe-Data_30-Oct-2019.xlsx

Boyle, T., Bradley, C., Chalk, P., Jones, R. y Pickard, P. (2003). Usar el aprendizaje combinado para mejorar las tasas de éxito de los estudiantes al aprender a programar. Revista de medios educativos, 28(2-3), 165-178. Recuperado de https://www.researchgate.net/publication/228775772_Using_Blended_Learning_to_Improve_Student_ Success_Rates_in_Learning_to_Program

Conrads, J., Rasmussen, M., Winters, N., Geniet, A. y Langer, L. (2017). Digital Education Policies in Europe and Beyond: Key Design Principles for More Effective Policies [JRC Science for Policy Report, JRC109311]. European Union. https://doi.org/10.2760/462941 
GAPP. Nueva Época - N. ${ }^{\circ}$ 26, julio 2021 - ISSN: 1989-8991 - DOI: https://doi.org/10.24965/gapp.i26.10831 - [Págs. 61-80]

El derecho a la educación y la seguridad en tiempos de Covid-19: Factores claves para la adopción de modelos de blended learning...

Amaya Gil Albarova / Cristina Monge Lasierra / Amparo Gracia Bernal / Federico Buyolo García

Ministerio de educacion y formación profesional (MEFP) (2020). Estadísticas de la Educación. https://www. educacionyfp.gob.es/servicios-al-ciudadano/estadisticas.html

Ferrer, F., Belvís, E. y Pàmies, J. (2010). Tablet PCs, academic results and educational inequalities. Computers \& Education, 56(1), 280-288. https://doi.org/10.1016/j.compedu.2010.07.018

Fidalgo-Blanco, A., Sein-Echaluce, M. L. y García-Peñalvo, F. C. (16 de enero de 2020). Ventajas reales en la aplicación del método de Aula Invertida-Flipped Classroom (version 1). Zenodo. http://doi.org/10.5281/ zenodo.3610578

Fundación COTEC (20 de abril de 2020a). Propuestas para cinco posibles escenarios educativos ante la crisis sanitaria del covid-19. https://cotec.es/cotec-publica-un-documento-con-propuestas-para-cinco-posibles-escenarioseducativos-ante-la-crisis-sanitaria-del-covid-19/

Fundación COTEC (2020b, 20 de abril). COVID-19 y Educación I: problemas, respuestas y escenarios. https://online. flippingbook.com/view/967738/

García-Ruiz, R., Aguaded, I. y Bartolomé, A. (2018). La revolución del blended learning en la educación a distancia. RIED. Revista Iberoamericana de Educación a Distancia, 21(1), 25-32. http://dx.doi.org/10.5944/ried.21.1.19803

Garrison, D. R. y Kanuka, H. (2004). Blended learning: Uncovering its transformative potential in higher education. The internet and higher education, 7(2), 95-105. https://doi.org/10.1016/j.iheduc.2004.02.001

González-Patiño, J. y Poveda, D. (2015). Privileging the Individual Through the Collective Commitment: Parental Strategies and Dynamics Of Involvement in a Middle-ClassSchool. Multidisciplinary Journal of Educational Research, 5(3), 316-336. https://doi.org/doi:10.17583/remie.2015.1524

Harris, W. J. y Smith, L. (2004). Laptop use by seventh grade students with disabilities: Perceptions of special education teachers. Education Technology, 14. https://digitalcommons.usm.maine.edu/cepare_technology/14/

Hart, J. (2019). Top Tools for Learning [Results of the 13th Annual Learning Tools Survey]. Centre for Learning \& Performance Technologies. Recuperado de https://www.toptools4learning.com/

Hinojo, M. y Fernández, A. (2012). El aprendizaje semipresencial o virtual: nueva metodología de aprendizaje en Educación Superior. Revista Latinoamericana de Ciencias Sociales, Niñez y Juventud, 10(1), 159-167. http:// revistaumanizales.cinde.org.co/rlcsnj/index.php/Revista-Latinoamericana/article/view/591

Impacto de las TIC en las escuelas europeas (2020). Eduteca. http://eduteka.icesi.edu.co/articulos/ICT_InformeEuropa

Inamorato dos Santos, A., Punie, Y. y Castaño-Muñoz, J. (2016). Opening up Education: a Support Framework for Higher Education Institutions [JRC Science for Policy Report] (EUR 27938 EN). European Union. https:// op.europa.eu/en/publication-detail/-/publication/c52b6cab-a82c-4e75-8420-d2431196d11d/language-en

INE (2019). Las nuevas tecnologías en los hogares. Public tableau. Recuperado de https://public.tableau.com/views/ ticPrueba/His_TIC_es?:showVizHome=no\&:embed=true

Instituto Nacional de Cálidad y Evaluación - INCE (2000). Sistema estatal de indicadores de la evaluación (pp. 126127). Ministerio de Educación y Cultura. https://www.educacionyfp.gob.es/dctm/ievaluacion/indicadoreseducativos/ind2000.pdf?documentld=0901e72b8011107e

Kampylis, P., Punie, Y. y Devine, J. (2015). Promoción de un aprendizaje eficaz en la era digital. Un marco europeo para organizaciones educativas digitalmente competentes [Ciencia para la política, informe del JCR] (EUR 27599 ES). Instituto Nacional de Tecnologías Educativas y de Formación del Profesorado (INTEF). Ministerio de Educación, Cultura y Deporte. https://sede.educacion.gob.es/publiventa/promocion-de-un-aprendizaje-eficaz-en-la-era-digital-unmarco-europeo-para-organizaciones-educativas-digitalmente-competentes/ensenanza-recursos-digitales/21199

Kenney, J. y Newcombe, E. (2011). Adopting a Blended Learning Approach: Challenges Encountered and Lessons Learned in an Action Research Study. Journal of Asynchronous Learning Networks, 15(1), 45-57. https://files.eric. ed.gov/fulltext/EJ918218.pdf

Lei, J. y Zhao, Y. (2008). One-to-one computing: what does it bring to schools? Journal of Educational Computing Research, 39(2), 97-122. https://doi.org/10.2190/EC.39.2.a

Martinez Celorrio, X. (2020). Guía de actuaciones para la continuidad educativa y contra la brecha digital y de aprendizaje. Secretaria Federal de educación del PSOE. https://www.psoe.es/actualidad/documentos-de-interes/ guia-de-actuaciones-para-la-continuidad-educativa-y-contra-la-brecha-digital-y-de-aprendizaje/

Mackey, K. y Watson, J. (2015). Proof points: Blended learning success in school districts [entrada en blog]. Christensen Institute. https://www.christenseninstitute.org/publications/proof-points/

Noorani, S. y Croisier, D. (6 de mayo de 2020). Focus on: Can the coronavirus pandemic improve our schools? Eurydice. https://eacea.ec.europa.eu/national-policies/eurydice/content/focus-can-coronavirus-pandemic-improve-our-schools_en

Redecker, C. y Punie, Y. (2017). European Framework for the Digital Competence of Educators: DigCompEdu [JRC Science for Policy Report] (EUR 28775 EN). Oficina de Publicaciones de la Unión Europea. https://doi. org/10.2760/159770

Sanz, I., Sáinz González, J. y Capilla, A. (2020). Efectos de la crisis de coronavirus en la educación. Organización de Estados Iberoamericanos para la Educación, la Ciencia y la Cultura - OEl. https://oei.int/oficinas/secretariageneral/publicaciones/efectos-de-la-crisis-del-coronavirus-en-la-educacion

Siemens, G., Gasevic, D. y Dawson, S. (2015). Preparing for the digital University: a review of the history and current state of distance, blended and online learning. Athabasca University Press. http://linkresearchlab.org/ PreparingDigitalUniversity.pdf 
GAPP. Nueva Época - N. ${ }^{\circ} 26$, julio 2021 - ISSN: 1989-8991 - DOI: https://doi.org/10.24965/gapp.i26.10831 - [Págs. 61-80]

El derecho a la educación y la seguridad en tiempos de Covid-19: Factores claves para la adopción de modelos de blended learning...

Amaya Gil Albarova / Cristina Monge Lasierra / Amparo Gracia Bernal / Federico Buyolo García

Staker, H. (2011). The Rise of K-12 Blended Learning. Profiles of emerging mode/s. Instituto Innosight. https://eric. ed.gov/?id=ED535181

Staker, H. y Horn, M. B. (2012). Classifying K-12 Blended Learning. Innosight Institute. https://files.eric.ed.gov/fulltext/ ED535180.pdf

Stein, J. y Graham, C. R. (2013). Essentials for Blended Learning. A Standards-Based Guide. Routledge. https://doi. org/10.4324/9780203075258

Desglosar el objetivo de Desarrollo Sostenible 4 - Educación 2030 (2016). UNESCO. https://unesdoc.unesco.org/ ark:/48223/pf0000246300_spa

Volman, M., Van Eck, E., Heemskerk, I. y Kuiper, E. (2005). New technologies, new differences. Gender and ethnic differences in pupil's use of ICT in primary and secondary education. Computers \& Education, 45(1), 35-55. https:// doi.org/10.1016/j.compedu.2004.03.001 